\title{
Analysis and Forecasting of Geomagnetic Field Signal in Active Period
}

\author{
Dingxin CHEN ${ }^{1, a,{ }_{*}}$, Daizhi LIU ${ }^{1}$, Liang Meng ${ }^{1}$, Yihong $\mathrm{LI}^{1}$, Chao NIU ${ }^{1}$ \\ ${ }^{1}$ Xi'an Research Inst. of Hi-Tech, Xi'an,710025, China \\ a chendx12@mails.tsinghua.edu.cn
}

\begin{abstract}
Keywords: geomagnetic variation field; active period; artificial neural network; modeling and forecasting.

Abstract. Geomagnetic variation is divided into quiet period and active period, while active period is non-periodic and random. This paper utilizes analyze the magnetic variation field signals in active period in time-frequency domain, then models and forecasts the signals by using Artificial Neural Networks. The results show that the 4-hour-forecasting error of the three methods is $M A E \leq 2.3 n T$. In multi-step forecasting, the method of GRNN is smooth, while LNN causes error increasing apparently. RBFNN has the best performance as its MAE is the smallest one for each time.
\end{abstract}

\section{Introduction}

Raised from the the magnetosphere-ionosphere current system and the induction current in the earth[1], Geomagnetic variation field is tightly connected to the activity of the sun, magnetosphere and ionosphere[2], and has extensive impact in the fields of space weather forecasting[3] and geomagnetic navigation[4,5]. The modeling and forecasting of geomagnetic variation field, which has effect in the accuracy of space weather forecasting and geomagnetic navigation, is of importance in application[5, 6].

The nonlinear interaction between various parameters in geomagnetic dynamic system makes the attempt to use a complete theoretical model to describe the magnetic field changes and further modeling forecasting possible [6].He Kang et al used PWVD method to analyze the data of geomagnetic $\mathrm{Z}$ component before Wenchuan Earthquake to get the time-frequency analysis, and detected magnetic anomaly signal [7]; Niu Chao et al utilized EEMD method to separate signal, modeled and forecasted geomagnetic field signal with improved ELMAN neural network [8], then they proposed the MEEMD - sample entropy - LSSVM model, made the 24h multi-step forecasting error to 3.0nT[9].

Geomagnetic variation is divided into quiet period and active period. Quiet period is composed of periodic and regular changes, while active period is non-periodic and random.[2] Based on the result of time frequency analysis, this paper focused on the predictability of geomagnetic signal in active period.

\footnotetext{
Analysis

The severe degree of geomagnetic variation is effected by ionosphere activity, solar wind and other factors, then directly influence the accuracy of forecasting. The Z-component is used as the matching parameter in application, therefore we make Z-component the object to model. The data used in experiment is 10min-mean-data of geomagnetic variation field form Chinese QIX station, 2009. As is shown in Fig. 1, the Z-component in 25-26 week (2009.06.19-2009.07.02), with high value of $K_{p}$ index, is relatively active.
} 

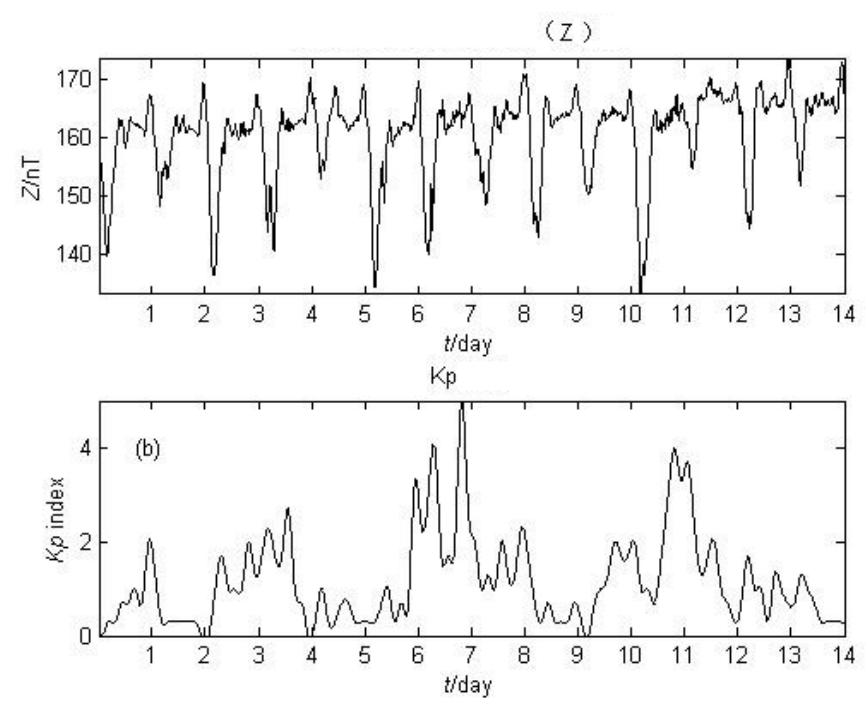

Fig. 1: Z-component and index of data from QIX station of Chinese Geomagnetic Network The time-frequency distributions of signals in different periods are shown in Fig.2, which has obvious change with time. The signal is of typical non-stationary characteristics, and has more complex components. Although the average energy of the disturbance components is not big all through the time, they could form a large peak at some time points. This is why it is hard to analyze and forecast the geomagnetic variation field in active period.

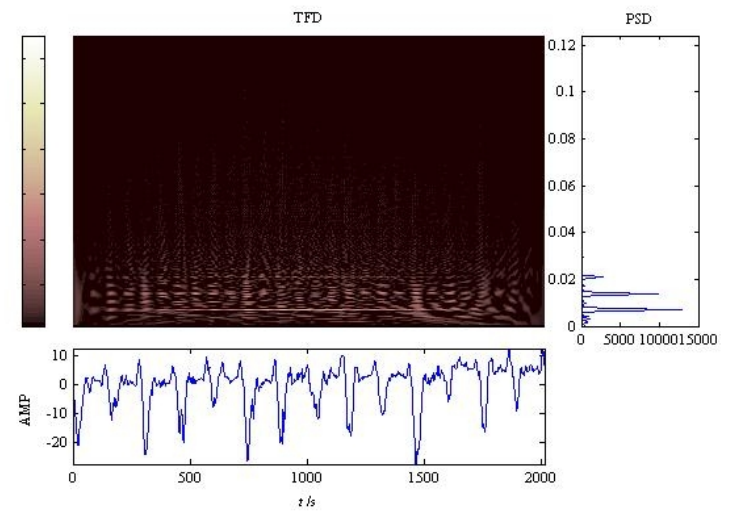

Fig. 2: Wigner-Ville distribution of $\mathrm{Z}$ component signal

\section{Modeling and Forecasting}

As to the signal of active period, we choose Linear Neural Network (LNN), Radical Basis Function Neural Network (RBFNN), General Regression Neural Network (GRNN) to analyze the geomagnetic field.

\section{Linear Neural Network (LNN) Forecasting}

LNN is composed of one neuron or more, whose activation functions are linear function.[14] LNN could directly calculate the network's weights and thresholds without training. If the network has more than one zero-error solutions, then it takes the smallest one for weights and thresholds. In the case of nonexistence of zero-error solution, LNN accords to the Least Mean-Square (LMS) to selects weights and thresholds. When analyzing the signal at the time $t$, we take the geomagnetic field signal since 1 week before as the sample to get the training models, and then utilize these models to forecast the value at time point $t$. Take $t \in[1009,2016]$, the results of 2 hour multi-step forecasting are shown in Fig.3. 

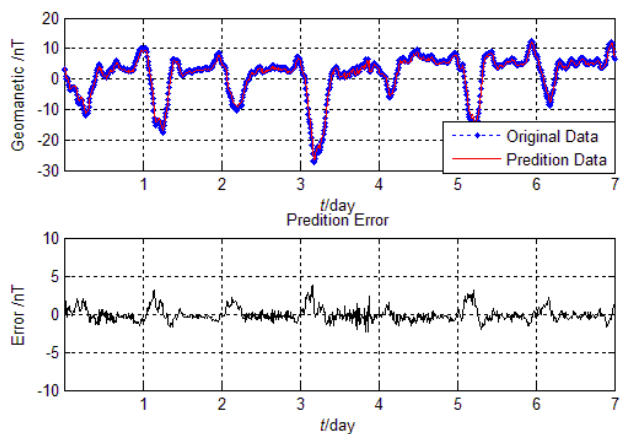

Fig.3 LNN multi-step forecasting of $Z$ component signal (2h)

As is shown in Fig.3, LNN could trace the signal well, although it causes huge errors in the extreme points.

\section{Radical Basis Function Neural Network (RBFNN) Forecasting}

RBFNN is a three layer forward network, consisted of three parts: a set of sensing unit (source node) of input layer, hidden layer of the computing nodes and input layer of the computing nodes .Input signal to the hidden layer, the node function is Gaussian Function. Output layer node function is usually a linear function. Take spread $=100, t \in[1009,2016]$, the results of 2 hour multi-step forecasting are shown in Fig.4.
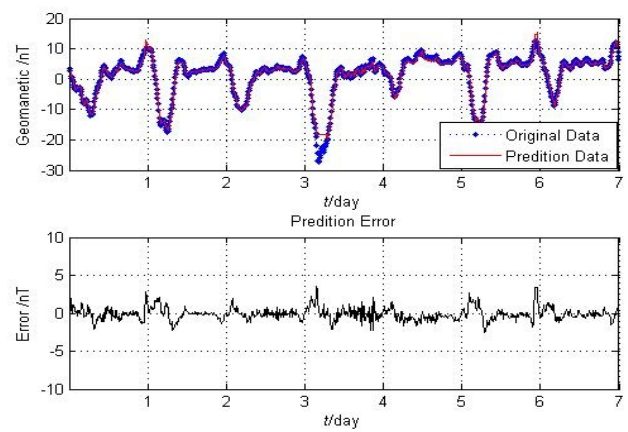

Fig.4 RBFNN multi-step forecasting of $\mathrm{Z}$ component signal (2h)

\section{General Regression Neural Network (GRNN) Forecasting}

GRNN, a feedforward neural network based on the theory of nonlinear regression analysis, is a variation form of RBFNN. It activates neurons to trace function, and is composed of input layer, hidden layer and output layer. Hidden layer uses Gauss Transformation Function to control the input, each training vector in the hidden layer has a corresponding training RBF neuron, and stored in each hidden layer neuron. GRNN doesn't change weights between neurons in training, to make sure network learning entirely depends on data samples, so GRNN avoids the influence of the subjective assumption to the forecasting. Taket $\in[1009,2016]$, the results of 2 hour multi-step forecasting are shown in Fig.5. 

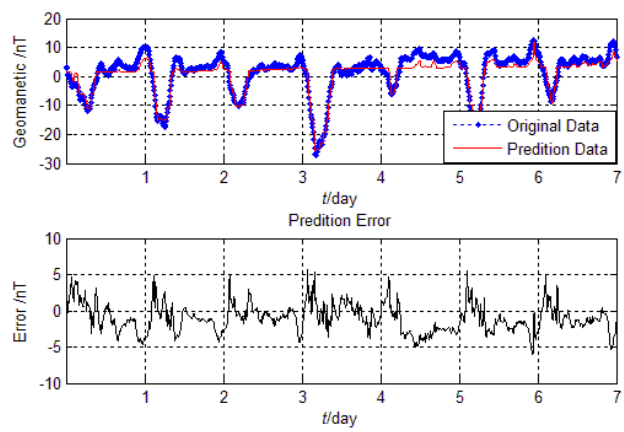

Fig.5 GRNN multi-step forecasting of $\mathrm{Z}$ component signal (2h)

\section{Result}

This paper take the 1-week long 10-minites-mean Z-component geomagnetic field signal sequence as train data, and forecasting time interval 0 24h. Mean Absolute Error (MAE) of the multi-step forecasting results are shown in Fig.6. Just as expected, MAE rises as forecasting time extends, because of accumulation error in the process of iteration. As is accorded in Table1, GRNN has relatively jarless result in multi-step forecasting examinations, as MAE chould be concentrated on some value when forecasting time longer than $4 \mathrm{~h}$. RBFNN has the best performance among the 3 methods. As time gets longer, LNN cause MAE rise apparently. All the 3 methods could control the forecasting error in the interval of $M A E \leq 2.3 n T$.

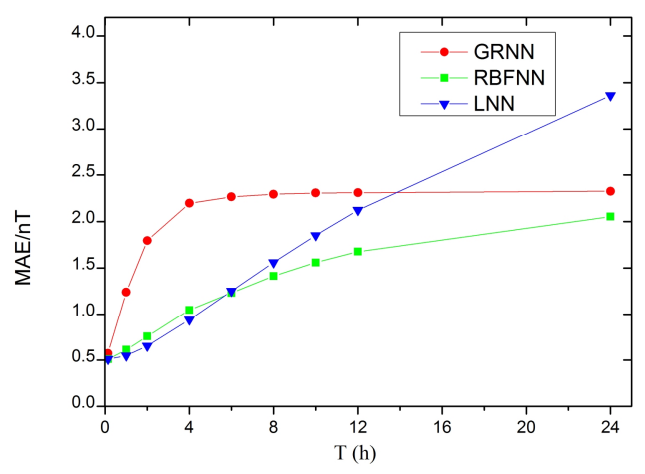

Fig.6 MAE of multi-step forecasting of geomagnetic sigals

Table1 MAE of multi-step forecasting

\begin{tabular}{cccccccccc}
\hline Time & $10 \mathrm{~m}$ & $1 \mathrm{~h}$ & $2 \mathrm{~h}$ & $4 \mathrm{~h}$ & $6 \mathrm{~h}$ & $8 \mathrm{~h}$ & $10 \mathrm{~h}$ & $12 \mathrm{~h}$ & $24 \mathrm{~h}$ \\
\hline LNN & 0.51152 & 0.54856 & 0.65332 & 0.93924 & 1.25070 & 1.55899 & 1.85131 & 2.12237 & 3.36225 \\
\hline RBFNN & 0.50970 & 0.61326 & 0.75871 & 1.04702 & 1.23156 & 1.41255 & 1.55748 & 1.67563 & 2.05241 \\
\hline GRNN & 0.57376 & 1.23908 & 1.7948 & 2.19673 & 2.26540 & 2.29387 & 2.30756 & 2.31013 & 2.32533 \\
\hline
\end{tabular}

\section{Conclusion}

This paper in view of the active period geomagnetic station signal, analyzed characteristic using the time-frequency, modeled and forecasted in three kinds of neural networks, separately. In the process of multi-step forecasting, GRNN and RBFNN perform well, little influence occurs in the precision of multi-step prediction, while LNN increase MAE as the forecast time extends. RBFNN's MAE value is minimum. 


\section{Acknowledgement}

This work is supported by the National Natural Science Foundation of China (Grant No. 41374154).

\section{Reference}

[1] Xu Wenyao. Geomagnetism[M]. Beijing: Seismological Press, 2003:35.

[2] Yuan Yanghui. Study of Geomagnetic Variation Field in The Geomagnetic Navigation[D]. Huazhong University of Science and Technology, 2012: 18-19.

[3] $\mathrm{Xu}$ Wenyao. Physics of Electromagnetic Phenomena of The Earth[M]. Hefei: University of Science and Technology of China Press.2009:473-474.

[4] Xu Wenyao. The Improvement of Numerical Calculation and Determination Method of Index K in Geomagnetic Activity[J]. Northwestern Seismological Journal, 2005, 27(S): 36-41.

[5] Guo Caifa, Zhang lijun, Cai hong. Analysis of geomagnetic navigation accuracy under magnetic storms. Chin. J Space Sci. , 2001, 31(3): 372-377.

[6] Zhao Ming, Zeng Xiaoping, Lin Yunfang. Nonlinear Analysis of Magnetic Storm In March 13, 1989[J]. Observation and Research on Seismological and Geomagnetism, 1997, 18(3): 56-60.

[7] He Kang, Yan Rui, Zheng Haigang, etc. The Application of Pseudo WVD in Geomagnetic Time-frequency Analysis[J]. China's Seismic Research, 29(1): 157.

[8] Niu Chao, Lu Shikun, Qi Shufeng. Prediction of the Geomagnetic Variation Field Based on Modified Ensemble Empirical Mode Decomposition and Modified Elman Neural Network[J]. Journal of Heibei University/Natural Science Edition, 2014, 38(1): 50-54.

[9] Niu Chao, Li Xihai, Liu Daizhi, etc.. Forecasting Model of Geomagnetic Variation Field Based on Modified Ensemble Empirical Mode Decomposition-Sample Entropy-Least Square Support Vector Machine[J]. Geomatics and Information Science of Wuhan University, 2014, 39(5): 626-630. 